\title{
Évaluer la durabilité des systèmes et des territoires aquacoles : proposition méthodologique
}

Eduardo Chia $^{1}$

Hélène Rey-Valette ${ }^{2}$

Jérôme Lazard ${ }^{3}$

Olivier Clément ${ }^{4}$

Syndhia Mathé ${ }^{2}$

1 Inra/Cirad

UMR Innovation

2, place Viala

34000 Montpellier

France

<chia@supagro.inra.fr>

2 Université de Montpellier I

Faculté de Sciences Économiques

Avenue de la Mer, CS 79606

34960 Montpellier cedex 2

<helene.rey-valette@univ-montp1.fr>

<syndhia.mathe@univ-montp1.fr>

${ }^{3}$ Cirad UR "Aquaculture et gestion

des ressources aquatiques"

TA B-20/01

Avenue Agropolis

34398 Montpellier cedex 5

France

<jerome.lazard@cirad.fr>

${ }^{4}$ Inra

UMR NuAGe

Pôle d'hydrobiologie

Quartier Ibarron

64310 Saint-Pée-sur-Nivelle

France

<clement@st-pee.inra.fr>

\begin{abstract}
Résumé
En devenant une référence, une contrainte, une action performative pour l'ensemble des activités économiques, le développement durable transforme non seulement les pratiques productives et organisationnelles, mais il produit également de nouveaux objets et situations de recherche qui nécessite des renouvellements méthodologiques. À l'occasion d'un programme de recherche sur l'élaboration d'une démarche d'évaluation du développement durable dans l'aquaculture, un protocole méthodologique a été élaboré de façon interdisciplinaire et en prenant en compte les représentations des acteurs. Il permet notamment de rendre compte de l'insertion territoriale et institutionnelle des systèmes aquacoles. La logique de la démarche de coconstruction qui est menée, partant des principes pour identifier les critères puis les indicateurs, comporte une dimension générique de façon à pouvoir être appliquée à d'autres systèmes aquacoles, voire à d'autres secteurs. Après avoir présenté les hypothèses et postulats qui fondent la démarche, les auteurs présentent le programme de recherche en insistant sur les aspects méthodologiques. En conclusion, ils discutent de l'intérêt et des modalités de généralisation de la méthode, ainsi que du statut de la relation construite avec les acteurs à cette occasion.
\end{abstract}

Mots clés : aquaculture ; coconstruction ; développement durable ; indicateur écologique ; interdisciplinarité ; méthodologie.

Thèmes : méthode et outil ; pêche et aquaculture ; productions animales.

\section{Abstract \\ Approach for evaluating sustainable development in aquaculture: A methodological protocol}

In becoming a reference, a constraint, a high performance action for all economic activities, sustainable development transforms not only productive and organisational practices but equally produces new subjects and research situations that require methodological renewal. On the event of a research programme on the elaboration of an evaluation approach of sustainable development in aquaculture, a methodological protocol was elaborated in a multidisciplinary manner while taking into consideration the representations of actors. This protocol also makes it possible to give an account of the territorial and institutional insertion (introduction) of fish farming systems. The logic of the coconstruction approach that is carried out, starting from the principles to identify the criteria and indicators, constitutes a standard dimension which could be applied to other fish farming systems and even to other sectors. After presenting the hypothesis and assumptions that justify the approach, the actors present the research programme while emphasising methodological aspects. In conclusion, they debate the importance and terms of generalising the method as well as the types of relationship built with the actors in this event.

Key words: aquaculture; coconstruction; ecological indicators; interdisciplinarity; methodology; sustainable development.

Subjects: animal production; fishing and aquaculture; tools and methods.

Tirés à part : J. Lazard 
a nature du développement durable, comme système de valeur ou métanorme (Offredi, 2006), implique un processus de changement qui nécessite une évolution progressive, du fait des conditions spécifiques de modification des métarègles (Aoki, 2006). Ce changement doit s'accompagner d'un processus de traduction (Rudlof, 2006) pour assurer une relative familiarité avec des savoirs communs et faciliter l'appropriation du nouveau référentiel. Ainsi, la mise en ouvre du développement durable doit être pensée comme un processus d'hybridation "bricolé " et flexible. Cette mise en œuvre est, actuellement, au coeur des politiques publiques (agendas 21) et des stratégies d'entreprises (responsabilité sociale des entreprises). Elle concerne aussi, en premier plan, la recherche, nécessitant un renouvellement à la fois des domaines de production, de connaissance et des pratiques de recherche (Godard et Hubert, 2002). En effet, comme le rappellent Boulanger et Bréchet (2003), l'article 35 de l'Action 21 de Rio signale la nécessité d'adapter les outils analytiques en appui au développement durable de façon à prendre en compte ses spécificités en matière d'interdisciplinarité, d'intergénérationalité, d'incertitude, d'articulation entre échelles locale et globale et de participation des acteurs. Il apparaît donc qu'il crée de nouveaux objets et situations de recherche pour lesquels la communauté scientifique n'est pas nécessairement bien armée, en particulier en matière d'évaluation. Celle-ci est souvent vue par les acteurs comme l'affaire des chercheurs, de l'administration ou des experts internationaux. Il s'agit dans ce cas-là de démarches plutôt normatives et top down, initiées, le plus souvent, par la recherche ou les institutions internationales (avec ou sans les acteurs), à l'occasion d'analyses d'impact ou pour répondre aux exigences d'évaluation finale des projets et des politiques. Les finalités sont alors plutôt réglementaires et souvent coercitives. Le processus d'appropriation des indicateurs par les acteurs, pour guider leur action, devient alors difficile, voire impossible. Or, cette appropriation constitue une condition de la fonctionnalité et de la pérennité des systèmes d'observation (Reix, 2007), sous peine de renforcer les "cimetières " d'indicateurs et d'observatoires. Ainsi, bien qu'on observe un foisonnement d'initiatives d'élaboration d'indicateurs de développement durable (Rey-Valette et al., 2007), force est de constater qu'il ne fait l'objet que d'une faible appropriation par les acteurs, ou, au contraire pour certains, le développement de routines défensives (Argyris et Schön, 1996).

Dans ce contexte, à l'occasion d'un programme de recherche sur " l'évaluation de la durabilité des systèmes aquacoles " (EVAD), nous avons élaboré un protocole méthodologique d'évaluation de la durabilité fondé sur une démarche de coconstruction d'indicateurs de développement durable pour les systèmes aquacoles. La démarche proposée est fondée sur une approche territorialisée du développement durable. Elle s'appuie sur des recherches de terrain menées dans cinq pays (Cameroun, Chypre, France - Bretagne et Méditerranée -, Indonésie et Philippines) correspondant à des contextes ruraux et littoraux et à des systèmes d'élevage diversifiés (Lazard et al., 2009). Cette dimension territoriale est fondamentale pour l'appropriation et l'institutionnalisation du développement durable du fait des conditions de décentralisation des décisions qu'il suppose. Un grand nombre de travaux récents (Lazzeri, 2006) souligne l'intérêt des expériences de coconstruction locale d'indicateurs de développement durable. La prise en compte de la nature du développement durable comme référentiel et des enseignements récents quant aux apprentissages et processus d'appropriation spécifiques qui en résultent, nous conduit à fonder notre démarche sur le postulat selon lequel les aquaculteurs vont d'autant plus rapidement adhérer au développement durable et adopter les pratiques proposées pour sa mise en place d'autant plus rapidement que les représentations qu'ils se font du développement durable sont proches de celles qu'ils ont de leur activité. Les représentations étant un construit social, les aquaculteurs doivent se livrer à un travail de déconstructionreconstruction de ce qu'il convient de faire tant sur le plan individuel que collectif. Ce processus de déconstruction et de reconstruction suppose l'organisation d'une démarche réflexive ouverte à l'ensemble des parties prenantes et facilite l'émergence d'une culture commune du développement durable. Les recherches menées sur l'usage des indicateurs témoignent du fait que les actions de coconstruction d'indicateurs de durabilité constituent un cadre favorable à ce type de démarche réflexive (Rey-Valette et al., 2007b). Elles s'inscrivent dans la logique préconisée par Boiffin et al. (2004) " de la recherche des points critiques et des leviers d'actions par la mise au point de critères et méthodes d'évaluation". Ainsi, les indicateurs coconstruits devraient être plus utilisés du fait qu'ils ont acquis du sens pour les acteurs au travers de la démarche de coconstruction.

Après avoir présenté, dans une première partie, les spécificités du protocole méthodologique qui fondent la démarche, les auteurs présentent, dans une deuxième partie, les moments forts et modalités pratiques de la mise en œuvre de ce protocole qui a été adapté, affiné et validé à travers l'expérimentation faite dans le cadre du projet de recherche EVAD. La troisième partie détaille l'utilité du guide qui est produit et qui constitue le résultat du projet EVAD. En conclusion, les auteurs montrent que les relations construites avec les partenaires locaux à l'occasion du projet peuvent être assimilées à un partenariat d'exploration (Segrestin, 2003).

\section{Un protocole méthodologique basé sur une démarche systémique et participative}

Il ne s'agit pas de proposer des systèmes d'indicateurs clés en mains, ce qui serait contraire aux exigences du développement durable, mais une démarche, un guide pour l'élaboration d'indicateurs de durabilité et la mise en œuvre du développement durable dans l'aquaculture. Ce guide (Rey-Valette et al., 2008c) offre non seulement une check-list générique de principes, critères et indicateurs mais aussi, et surtout, un protocole méthodologique de coconstruction permettant aux utilisateurs de s'approprier la démarche de construction de leurs indicateurs et, par-là, de leur donner, collectivement et à leur échelle, du sens au développement durable.

Ce protocole a été conçu en intégrant les acquis des travaux sur l'usage des indicateurs et sur les conditions et modalités 
d'appropriation du développement durable. On considère donc que le développement durable exige un apprentissage collectif organisationnel en "double et simple boucle " (Argyris et Schön, 1996) qui implique une démarche participative de coconstruction d'indicateurs, pouvant conduire à un apprentissage collectif. Ainsi, ce protocole permet de tenir compte des représentations des parties prenantes et vise à favoriser leur participation.

La démarche méthodologique d'élaboration des indicateurs qui est proposée s'appuie sur le cadre " PCI : principes, critères, indicateurs " (Prabhu et al., 2000) partant des principes, pour définir les critères dont la mesure conduit à la définition des indicateurs. Ce type de démarche permet d'organiser la production d'indicateurs à partir non seulement des indicateurs existants, par ailleurs, et des normes qui visent à orienter l'implémentation du développement durable, mais aussi à partir des représentations des acteurs et des enjeux. On retrouve la logique dite prin- ciple guided, recommandée par Droz et Lavigne (2006), qui permet, en contextualisant les principes généraux du développement durable, de favoriser son appropriation par les acteurs.

La construction des indicateurs de développement durable peut relever de deux grands types de démarche soit descendante ou normative (top-down) lorsque l'indicateur résulte d'une représentation hiérarchisée du système en fonction des référentiels produits par des institutions extérieures, soit procédurale (bottom-up) lorsqu'il intègre une diversité de représentations au travers d'approches participatives. L'intérêt du protocole élaboré et expérimenté par le projet EVAD tient au fait qu'il réunit ces deux logiques en associant des phases de consultation et de mobilisation des acteurs et des phases de recherche, laquelle doit nécessairement être interdisciplinaire pour intégrer la pluralité des dimensions du développement durable. Il permet, ainsi, de bénéficier des atouts respectifs des deux logi- ques. En effet, si les démarches participatives offrent une plus grande légitimité et une plus grande adéquation des indicateurs produits aux enjeux locaux, leur caractère décentralisé et contextualisé génère des difficultés au niveau de l'intégration des acquis, de la comparaison à des échelles englobantes, ainsi qu'au niveau du financement de la pérennisation de ces démarches.

La figure 1 rend compte de l'organisation générale de la méthode qui distingue deux grandes phases: celle de la construction de la check-list, où les acteurs sont pris en compte à travers des enquêtes de terrain visant à identifier et à intégrer la diversité de leurs représentations, et celle de la validation de cette check-list. Il s'agit, au travers de groupes de travails collectifs rassemblant l'ensemble des parties prenantes, de hiérarchiser et de sélectionner les principes, critères et indicateurs produits de façon à identifier ceux qui font sens pour les acteurs et à révéler leurs préférences. Cette phase répond à

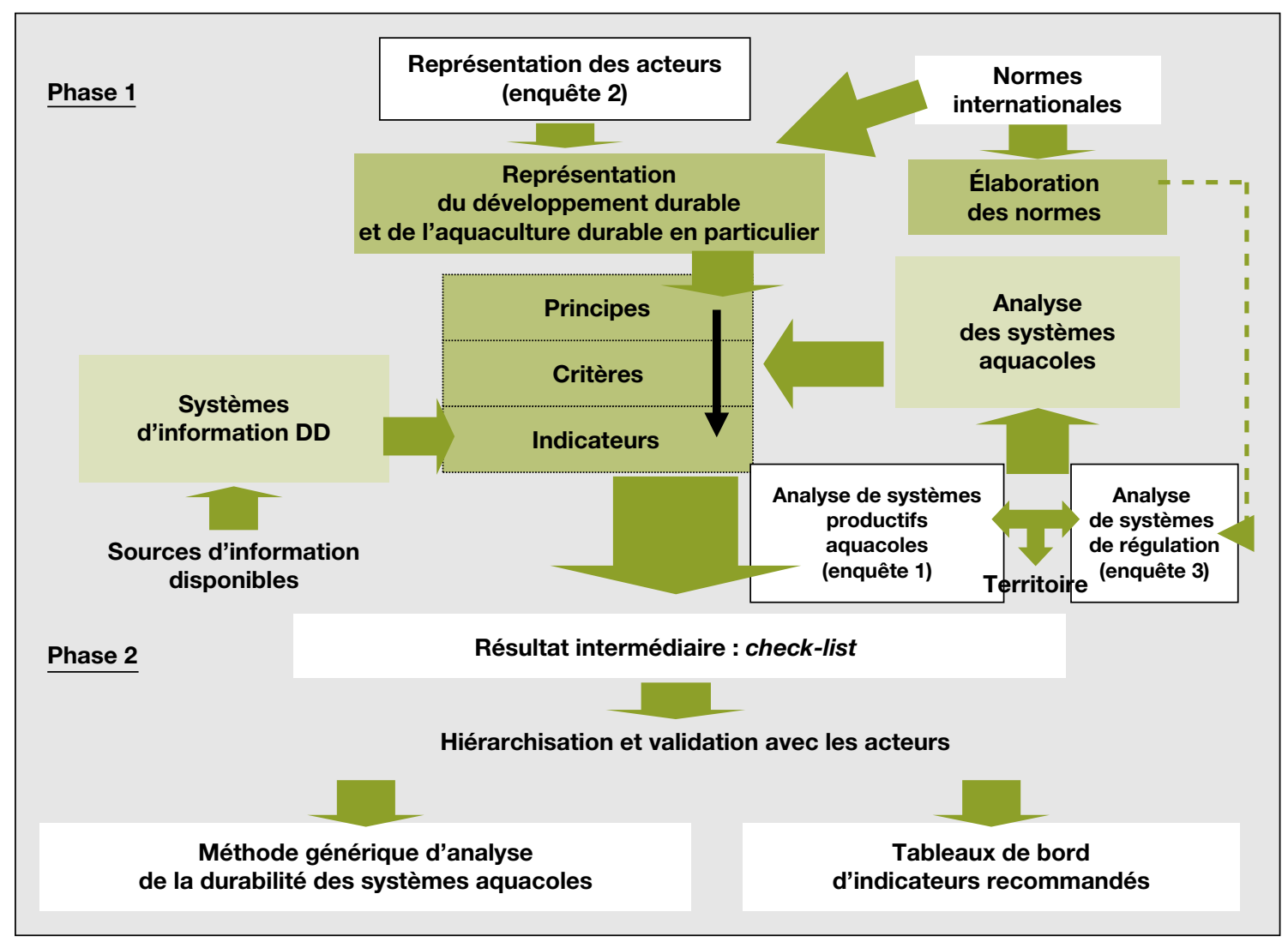

Figure 1. Présentation du protocole méthodologique structurant la démarche.

Figure 1. Presentation of the methodological protocol underlying the approach. 
des objectifs de formation, de communication et d'aide à la décision.

La première phase de la démarche comporte deux aspects clés, à savoir l'analyse des représentations des acteurs, qui suppose des enquêtes, et l'étude des systèmes aquacoles, qui nécessite une grille de lecture adéquate. En prolongeant les apports des recherches sur l'agriculture et l'élevage, nous avons, ainsi, établi un cadre d'analyse des systèmes aquacoles (Rey-Valette et al., 2008a) permettant, pour rendre compte du développement durable, d'articuler l'ensemble des facteurs tenant aux systèmes productifs, aux systèmes de régulation et au territoire (figure 2). La prise en compte de ces trois dimensions permet d'aborder la durabilité de deux points de vue complémentaires et interactifs : les facteurs de durabilité des exploitations aquacoles et la contribution des systèmes aquacoles à la durabilité des territoires qu'ils exploitent.

Les trois dimensions ainsi identifiées nécessitaient d'être explorées par des enquêtes spécifiques. Un ensemble de trois enquêtes coordonnées a été conçu pour étudier :

- le fonctionnement et les pratiques des aquaculteurs (enquête $\mathrm{n}^{\mathrm{O}} 1$ ) ;

- les représentations que les aquaculteurs et les parties prenantes ont du développement durable, de l'activité aquacole et des conséquences de l'application du développement durable à l'aquaculture (enquête $\mathrm{n}^{\circ} 2$ ) ;

- l'organisation des systèmes de régulation (enquête $\mathrm{n}^{\mathrm{O}} 3$ ).

La figure 3 rend compte de l'articulation de ces enquêtes et de leur contribution au protocole méthodologique d'identification des principes et critères de durabilité de l'aquaculture.

L'effectif des enquêtes a été établi en fonction de l'importance des systèmes aquacoles à l'échelle de chacun des terrains. Au total, pour l'ensemble des cinq pays 128 enquêtes relatives au fonctionnement des exploitations et aux pratiques des aquaculteurs (enquête $n^{\circ} 1$ ) et 168 enquêtes, visant à identifier les représentations des acteurs, ont été réalisées. Il s'agissait pour ces deux enquêtes, d'en- tretiens de face-à-face menés à partir de questionnaires et qui ont donné lieu à divers traitements statistiques d'analyse de données, ainsi que pour l'enquête sur les représentations à des analyses textuelles. Les enquêtes sur les systèmes de régulation ont, quant à elles, été menées par les partenaires locaux auprès de quelques acteurs clés. Il s'agissait d'entretiens non dirigés, structurés à partir d'une liste des types d'informations à recueillir.

Par ailleurs, signalons aussi que le projet comporte, en outre, une évaluation plus poussée des impacts environnementaux de l'aquaculture. Celle-ci est fondée sur des indicateurs synthétiques relevant de l'Analyse de cycle de vie (ACV) adaptée au secteur aquacole (Aubin et al., 2006) et appliquée sur chacun des terrains. Il s'agit d'une méthode d'analyse environnementale normalisée répondant aux normes internationales ISO (ISO, 2006a, 2006b). Son application à l'aquaculture a été conduite selon les préconisations de Guinée et al. (2002). Les traitements statistiques ont été réalisés avec le logiciel Simapro (Pré-consultant).

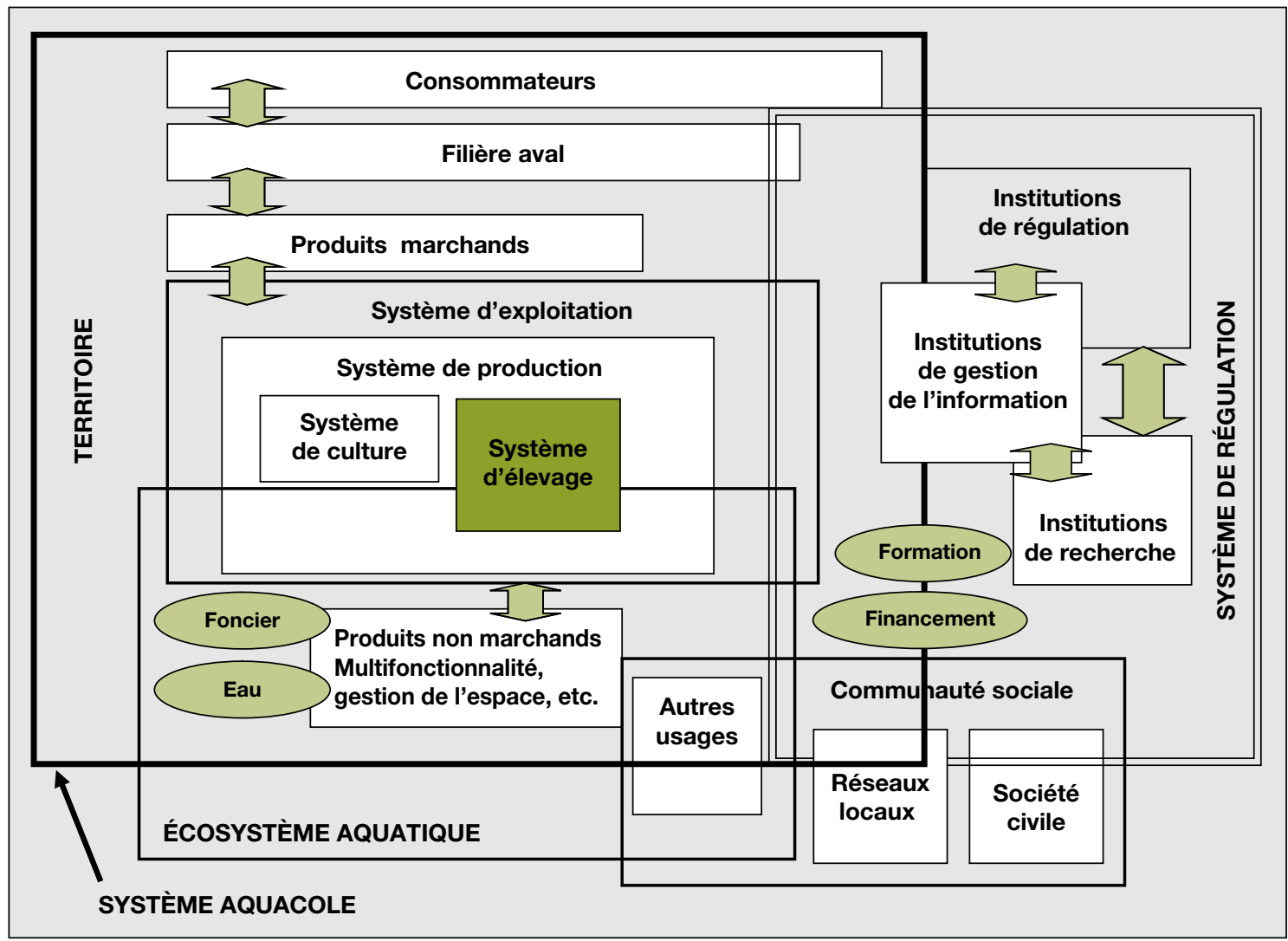

Figure 2. Présentation du cadre d'analyse systémique des systèmes aquacoles.

Figure 2. Presentation of the systemic framework of the fish farming systems. 


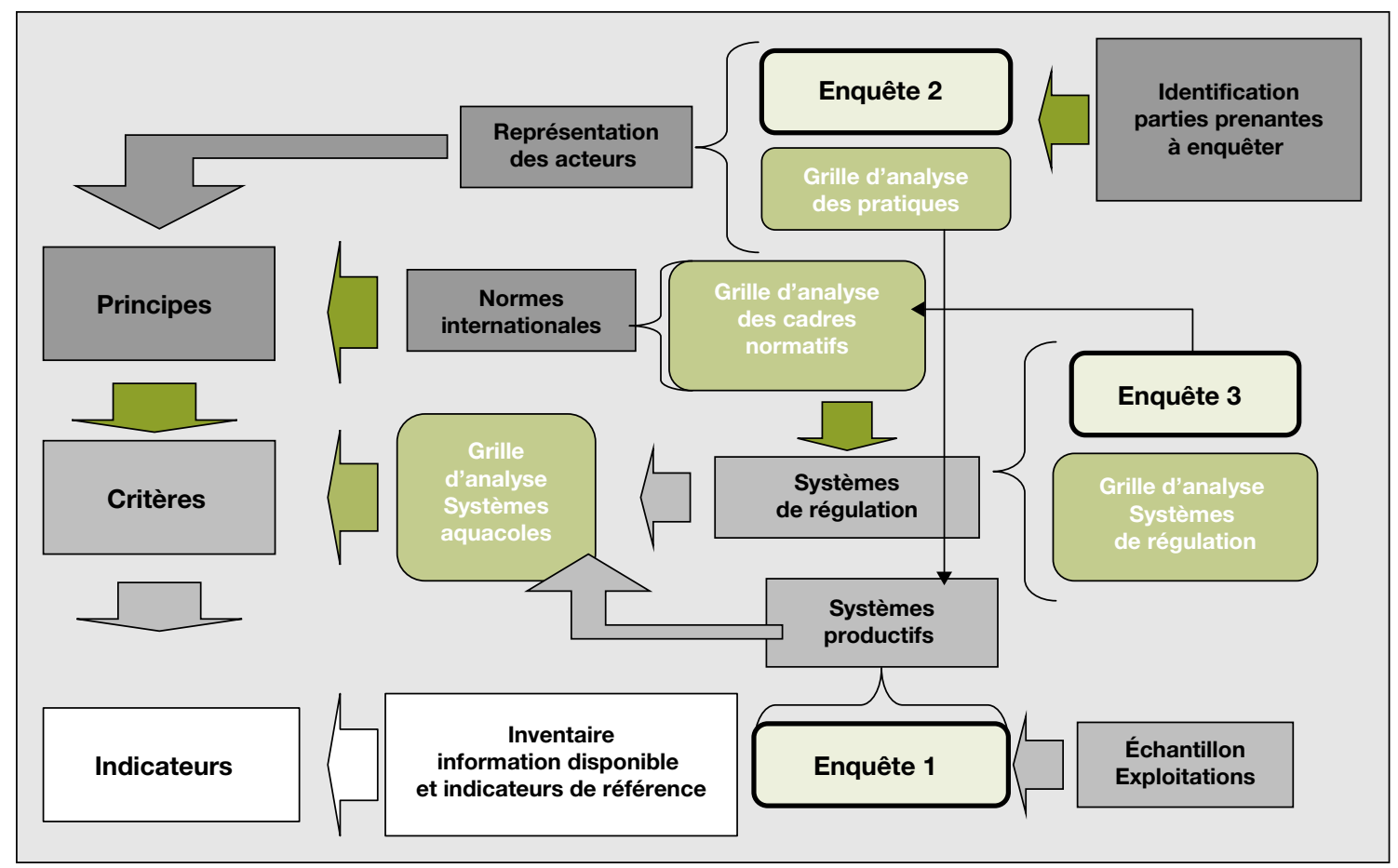

Figure 3. Articulation des enquêtes et structure méthodologique de la phase principes-critères-indicateurs.

Figure 3. Articulation of surveys and methodological structure of the Principles, Criteria and Indicators phases.

\section{Déroulement}

\section{et moments forts}

de la coconstruction

des indicateurs

de durabilité

des systèmes

\section{aquacoles}

\section{Identification des principes, critères et indicateurs à partir des représentations et des référentiels normatifs}

La phase d'identification des principes de développement durable constitue le point clé de la démarche. Ils sont issus des représentations de l'ensemble des parties prenantes des systèmes aquacoles (enquête $\mathrm{n}^{\circ} 2$ et pratiques observées à l'occasion de l'enquête $\mathrm{n}^{\mathrm{O}} 1$ ) et confrontés ensuite aux principaux référentiels existants et susceptibles d'être mobilisés par les acteurs (Rey-Valette et al., 2007a). La figure 4 tente de résumer les étapes de l'identification des principes de déve- loppement durable. Les 13 principes, ainsi formulés, sont présentés dans l'encadré 1.

À partir de ces principes, une check-list de critères et d'indicateurs a été élaborée de façon collective par l'équipe pluridisciplinaire des chercheurs en mobilisant :

- leurs connaissances des systèmes aquacoles des terrains étudiés ;

- les données de l'ensemble des enquêtes, de façon à identifier les variables clés des systèmes aquacoles tels que définis par la représentation systémique élaborée par le projet.

Deux types de variables et indicateurs ont été distingués selon qu'ils étaient relatifs à la durabilité des exploitations aquacoles ou à la contribution de l'activité aquacole à la durabilité des territoires, recouvrant ainsi l'emboîtement des deux échelles d'approches individuelle et collective de l'aquaculture et de son inscription territoriale.

\section{Étape de validation- hiérarchisation des PCI par les acteurs}

La démarche de validation-hiérarchisation et, par-là, la coconstruction du système d'évaluation de la durabilité de l'aquaculture, a été effectuée à l'échelle de chacun des terrains d'étude, de façon à mobiliser les acteurs locaux. Différents itinéraires ont été suivis selon les contextes (figure 5). Outre les chercheurs concernés par les différents terrains, les groupes de travail collectif rassemblaient, majoritairement, des aquaculteurs et des acteurs institutionnels (gestionnaires, organisations non gouvernementales), ainsi que des acteurs des filières économiques et quelques personnalités (tableau 1).

Les modalités de cette phase de validation ont été adaptées aux caractéristiques des terrains. Dans certains cas, l'ensemble de ce travail de validation a été réalisé dans le cadre de groupes de travail collectif tandis que dans d'autres cas, il a été l'occasion d'associer des entretiens bilatéraux (plus quelques contributions par courrier électronique pour les acteurs géographiquement trop distants) et des collectifs de travail. Hormis l'Indonésie où un atelier de jeux de rôle a structuré une partie du travail de validation, les groupes de travail collectif étaient organisés sous forme de débats visant à valider, collectivement à l'échelle de l'ensemble du système aquacole considéré, le résultat des hiérarchisations réalisées individuellement ou par sous-groupe.

Concernant les principes, ces hiérarchisations ont consisté, pour chacun des 


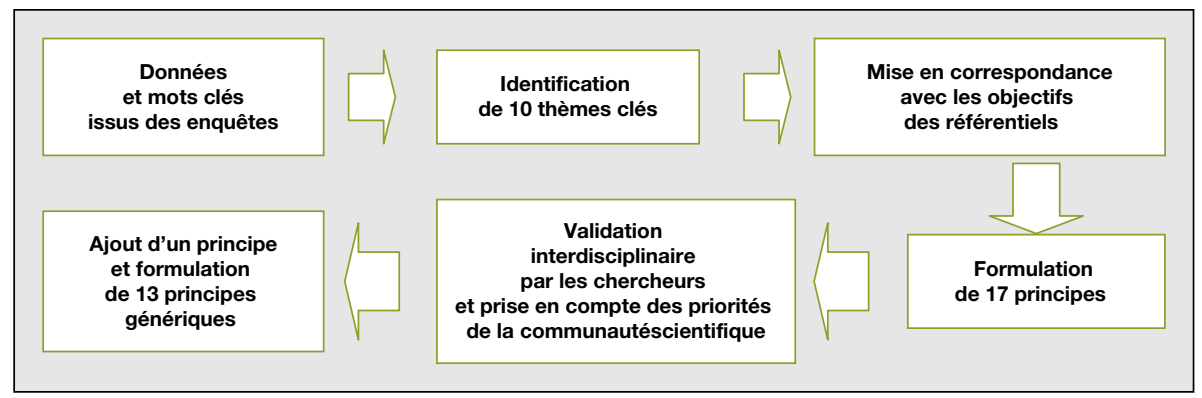

Figure 4. Traçabilité de la construction générique des principes de durabilité de l'aquaculture.

Figure 4. Traceability of the construction of standard principles of sustainable fish farming.

acteurs, à noter par ordre d'importance décroissante les dix principes jugés les plus importants au sein d'une liste provisoire qui en comportait 17 . Ceux-ci ont été réorganisés en 13 principes de référence, en fonction des résultats de la validation. Ainsi, le principe le plus important est noté 10 , tandis que le dernier de ceux sélectionnés obtient un score égal à 1 ; les sept restants se voyant attribuer un score nul. Ces notations individuelles ont permis d'établir des moyennes de score par principe, en distinguant les résultats par pays et par types d'acteurs. Dans un deuxième temps, pour chacun des prin- cipes sélectionnés, les acteurs ont évalué les critères en plusieurs catégories "prioritaire ", "important ", "à intégrer par la suite", "secondaire" ou "ne sais pas ", ce qui permet ensuite, en pondérant chaque catégorie par un coefficient ( 8 pour prioritaire, 4 pour important, 2 pour intégrer par la suite, 1 pour secondaire) de constituer aussi des moyennes par pays et par types d'acteurs.

Les discussions autour de la hiérarchisation-sélection des principes, critères et indicateurs ont constitué une démarche collective réflexive sur les enjeux et les modalités de l'aquaculture durable. Elle

\section{Encadré 1}

\section{Présentation des principes génériques de durabilité de l'aquaculture}

1. Contribuer à l'alimentation des sociétés

2. S'assurer de la protection des ressources naturelles et de l'adaptation à la capacité d'accueil des milieux

3. Améliorer le rendement écologique de l'activité (rendement énergétique, empreinte écologique, rendement protéiques)

4. Maintenir et valoriser la biodiversité ; respecter le bien-être animal

5. Faire reconnaître l'aquaculture comme une composante de l'aménagement du territoire

6. Renforcer la pérennité des exploitations

7. Renforcer la responsabilité sociale des entreprises aquacoles (conditions de travail, qualité de vie, etc.)

8. Développer des dispositifs pour des démarches de qualité, de certification, de labellisation de traçabilité visant à renforcer la qualité des produits

9. Mise en place de dispositifs institutionnels incitatifs permettant la participation des aquaculteurs et des parties prenantes en vue d'une meilleure adaptation des régulations

10. Renforcer la recherche et l'information relative au secteur

11. Accroître la capacité à faire face aux incertitudes et aux crises

12 Renforcer l'organisation et l'identité du secteur

13 Renforcer le rôle de l'État et des acteurs publics dans la mise en place du développement durable

a notamment permis de révéler les principes et critères qui faisaient le plus " sens " pour les acteurs et les motifs de rejet de ceux non sélectionnés. Cette procédure correspond à une vision négociée de ce que les acteurs considèrent comme étant le développement durable, la façon dont chacun peut et doit y contribuer et les règles qui permettent d'en "juger ". Les principes et critères qui sont sélectionnés doivent être suffisamment lisibles pour être compris par tous et pour faire l'objet d'une interprétation convergente. Ils doivent aussi pouvoir être aisément renseignés et stables de façon à constituer un système de pilotage des actions et permettre la mise en œuvre d'un suivi. Il s'agit d'une étape essentielle pour la construction d'un langage et d'un projet commun nécessaire à la mise en place du développement durable.

\section{Recherche finalisée et guide d'action conçu en vue d'une appropriation du développement durable}

Le guide qui est produit à l'issue du programme de recherche (Rey-Valette et al., 2008b) constitue un outil d'aide à la décision visant à accompagner les démarches sans se substituer aux acteurs, mais en cherchant, au contraire, à faciliter et à renforcer le processus d'appropriation du développement durable. À ce titre, il propose :

- des recommandations pratiques sur les modalités de mise en œuvre de la démarche d'élaboration d'indicateurs de durabilité de façon à s'inscrire dans une logique de coconstruction ;

- une check-list d'indicateurs de référence établie à partir d'une diversité importante de systèmes aquacoles et de territoires pour rechercher une relative exhaustivité des aspects abordés. La fonction générique de cette check-list restreint les indicateurs à leur fonction d'inventaire et constitue une base de référence facilitant les comparaisons ultérieures et relevant d'une sorte "d'objet statistique ou une synthèse de connais- 


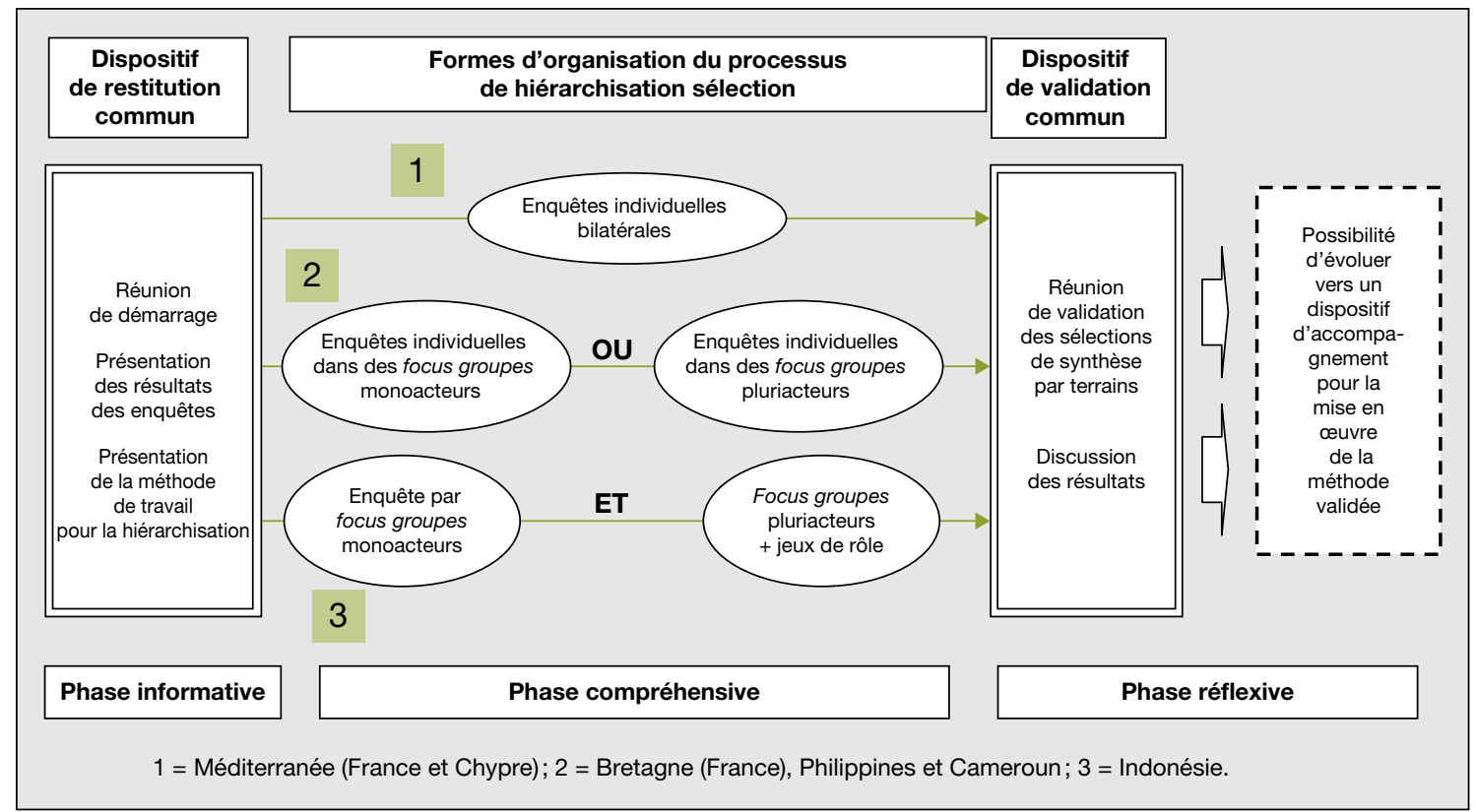

Figure 5. Présentation des protocoles suivis pour la phase de validation.

Figure 5. Presentation of validation framework.

sances intermédiaires permettant à la demande par des combinaisons multiples de produire une infinité de fonctions des éléments de cette liste initiale et ainsi de favoriser les démarches bottom-up de construction d'indicateurs " (Laloë, 2007).

Plus précisément, au niveau de l'aide à la décision, ce guide offre un appui différentié selon les moments du processus de mise en œuvre du développement durable. Trois types d'apports sont distingués en fonction des phases :

- lors des investigations préalables axées sur les questions de disponibilité d'information, face au foisonnement des référentiels et des observatoires déjà exis- tants à différentes échelles, le guide intervient comme un outil de gestion de l'information, en permettant de repérer et de classer les référentiels déjà existants en fonction d'une grille rendant compte du degré d'adéquation aux principes du développement durable (Mathé et al., 2006). En effet, le projet EVAD a inventorié et classé les cadres normatifs visant à promouvoir une aquaculture durable, ainsi que les initiatives de construction d'indicateurs existantes. Une base de métadonnées, facilitant l'accès à ces informations, a été construite. Elle permet de montrer une progression au cours du temps de l'adéquation des initiatives aux principes du développement durable tant au niveau des pratiques de construction que des objectifs poursuivis (Rey-Valette et al., 2007b) ;

- au niveau de la phase de mise en œuvre-appropriation, l'application de la démarche de coproduction d'un système de principes, critères et indicateurs favorise l'appropriation du développement durable et l'opérationnalité des indicateurs. En fonction des principes qui seront identifiés pour chaque application, la sélection d'indicateurs au sein de la check-list générique produite par le projet EVAD, du fait des correspondances établies avec d'autres référentiels dans le domaine de l'aquaculture (Consensus (UE, 2008), IDAqua (CIPA/ITAVI, 2007)),

\section{Tableau 1. Présentation des acteurs mobilisés pour la phase de sélection et de hiérarchisation.}

Table 1. Presentation of stakeholders mobilised for the hierarchical and selection phase.

\begin{tabular}{lcccccc}
\hline Type d'acteur & Bretagne & Cameroun & Indonésie & Méditerranée & Philippines & Total \\
\hline Acteurs hors du secteur productif & 8 & 21 & 27 & 21 & 16 & 93 \\
dont acteurs de l'administration & 8 & 15 & 27 & 8 & 16 & 74 \\
dont acteurs de la société civile & 0 & 6 & 0 & 13 & 0 & 19 \\
Acteurs du secteur productif & 9 & 17 & 30 & 13 & 26 & 95 \\
dont producteurs & 5 & 15 & 16 & 12 & 22 & 70 \\
dont acteurs de la filière & 4 & 2 & 14 & 1 & 4 & 25 \\
Total & $\mathbf{1 7}$ & $\mathbf{3 8}$ & $\mathbf{5 7}$ & $\mathbf{3 4}$ & $\mathbf{4 2}$ & $\mathbf{1 8 8}$
\end{tabular}


garantit la comparabilité d'un grand nombre des indicateurs proposés ;

- enfin, la dernière phase est relative à l'institutionnalisation du développement durable. Aggeri et al. (2005) distinguent trois formes possibles:

- la coercition, lorsque le changement de normes est imposé par une autorité hiérarchique ;

- le mimétisme, lorsqu'il relève d'un processus endogène ;

- la professionnalisation qui rend compte d'un processus volontaire interne à une communauté, lequel dépend alors des modes d'adoption et du niveau de structuration et de cohésion de cette communauté.

C'est de cette troisième logique que se réclame le projet EVAD par le caractère coconstruit du protocole proposé et par les apprentissages collectifs qu'il suppose au cours de cette coconstruction. En particulier, la prise en compte des représentations des parties prenantes constitue un apport essentiel à la mise en ouvre d'une gouvernance adaptée à la pérennisation des actions menées en faveur du développement durable. De même, l'évaluation de la contribution de l'aquaculture au développement durable des territoires permet d'offrir aux producteurs et associations professionnelles la possibilité de faire évoluer l'image de leur secteur et d'avoir des démarches proactives et des voies de dialogue avec les gestionnaires locaux. Il apparaît, en effet, que toute démarche de construction collective des indicateurs en tant "qu'outil " d'évaluation correspond à un processus décentralisé d'élaboration d'une nouvelle norme. Celui-ci peut constituer alors une ouverture et une opportunité pour les acteurs. Cependant, soulignons que pour participer efficacement et équitablement à ce processus, des innovations organisationnelles doivent être mises en œuvre, notamment de "professionnalisation" des organisations professionnelles et des collectifs d'acteurs (Aggeri et al., 2005).

\section{Conclusion}

Par son positionnement méthodologique, la visée de cet article était essentiellement de présenter une méthode de coconstruction des indicateurs de développement durable qui intègre les avancées scientifiques récentes quant aux conditions d'usage effectif des indicateurs et d'appropriation du développement durable par les acteurs. Testée de façon expérimentale dans le domaine de l'aquaculture à l'échelle de plusieurs sites représentatifs de la diversité technique, sociale et institutionnelle des systèmes aquacoles, elle ne peut pour l'instant permettre une évaluation sur le long terme des résultats en termes de participation des acteurs, d'opérationnalisation des systèmes de suivi et d'appropriation du développement durable. Toutefois, les applications expérimentales, qui ont été faites, montrent que sa mise en œuvre offre un cadre facilitateur augurant de son intérêt pour échanger et comparer les points de vue tant au sein des parties prenantes mobilisées qu'avec les chercheurs porteurs de la démarche. Elle a ainsi permis, à l'échelle des sites pilotes, d'initier des processus d'apprentissage collectif (Chia et al., 2008; Mathé et al., 2008) et de faire émerger des compromis autour de principes et critères communs en respectant un relatif équilibre entre les différentes dimensions du développement durable: environnementale, économique et sociale, mais aussi institutionnelle. L'introduction de cette dimension institutionnelle est une des originalités de la démarche qui permet de comprendre la capacité des acteurs à " inventer " de nouveaux dispositifs, arrangements ou règles. Répondant à la nécessité d'opérationnaliser les objectifs de participation du développement durable, cette dimension institutionnelle permet aussi d'intégrer l'analyse des dispositifs et modalités de gouvernance pouvant faciliter l'institutionnalisation des pistes collectivement définies, quant à la mise en œeuvre du développement durable. Elle conduit ainsi à articuler deux échelles interactives de la mise en œuvre locale du développement durable qui est celle des exploitations et celle de la contribution de ces exploitations au développement durale des territoires exploités. Dans le cas des applications expérimentales réalisées dans le cadre du projet EVAD, la coconstruction s'est appuyée sur des postures traditionnelles de recherche, et la mobilisation des acteurs s'est effectuée à partir d'enquêtes, de forums de discussions et de jeu de rôle dans le cas d'un des sites. Néanmoins, l'ensemble des échanges entre acteurs et chercheurs se sont révélés très fructueux et ont permis d'établir un climat de confiance, voire pour certains sites de révéler une demande de poursuite et d'approfondissement tant au niveau du suivi des indicateurs copro- duits que, plus largement concernant la définition de stratégies, en faveur d'une aquaculture durable. On peut donc rapprocher cette démarche de coconstruction, à une forme de " partenariat d'exploration" (Segrestin, 2003) qui peut constituer la première phase d'une collaboration plus étroite pouvant déboucher sur la mise en ouvre d'une rechercheaction pour accompagner la transformation des pratiques. Cependant, pour répondre aux exigences méthodologiques de toute recherche-action (Liu, 1997 ; Chia, 2004), il faudrait alors traduire l'intention de recherche et la volonté de changement en programme de travail et élaborer un cadre éthique où les engagements des uns et des autres seraient clairement indiqués. Pour ce faire, il faut prévoir la création d'un comité de pilotage de l'opération et d'un comité scientifique permettant une action réflexive sur la façon dont le travail est conduit et sur les types de connaissances créées. Rappelons en effet que l'objectif de toute recherche-action est double: résoudre les problèmes que se posent les acteurs et produire des connaissances scientifiques actionnables (Avenier et Schmitt, 2007).

\section{Remerciements}

Le projet de recherche EVAD (Évaluation de la durabilité des systèmes aquacoles) s'inscrit dans le cadre du programme fédérateur Agriculture et développement durable financé par l'Agence nationale de la recherche (ADD-ANR). Il associe cinq institutions de recherche (Cirad, Ifremer, Inra, IRD et université Montpellier I) et mobilise une équipe pluridisciplinaire d'une quinzaine de chercheurs (zootechniciens, biologistes, économistes, sciences de gestion). Les chercheurs du groupe méthodologique du projet qui rendent compte, ici, de la logique et de l'intérêt de la démarche tiennent à remercier et à associer à cet article les autres chercheurs de l'équipe, responsables des recherches sur les terrains, ainsi que du volet ACV (Analyse du cycle de vie) qui n'est pas détaillé dans cet article. Il s'agit par ordre alphabétique de : Joël Aubin (Inra Rennes, responsable du volet ACV), Jean-Paul Blancheton (Ifremer Palavasles-Flots), Marc Legendre (IRD Montpellier), Patrice Levang (IRD Indonésie), Olivier Mikolasek (Cirad Cameroun), Pierre Morissens (Cirad Montpellier) et François René (Ifremer Palavas-les-Flots). 


\section{Références}

Aggeri F, Pezet E, Abrassart C, Acquier A. Organiser le développement durable. Expériences des entreprises pionnières et formation de règles d'action collective. Paris: Vuibert ; Ademe, 2005.

Aoki M. Fondement d'une analyse institutionnelle comparée. Coll. Économie. Paris : Albin Michel, 2006.

Argyris C, Schön DA. Apprentissage organisationnel. Théorie, méthode, pratique. Bruxelles (Belgique) : De Boeck Université, 1996.

Aubin J, Papatryphon E, Van der Werf HMG, Petit J, Morvan YM. Characterisation of the environmental impact of a turbot (Scophthalmus maximus) recirculating production system using life cycle assessment. Aquaculture 2006 ; 261 : 1259-68.

Avenier M-J, Schmitt C. La construction des savoirs pour l'action. Coll. Action et Savoir/ Série rencontre. Paris : L'Harmattan, 2007.

Boiffin J, Hubert B, Durand N. Agriculture et développement durable. Enjeux et questions de recherche. Paris : Inra, 2004.

Boulanger P-M, Bréchet T. Modélisation et aide à la décision pour un développement durable : état de l'art et perspectives. Rapport final au SPP Politiques scientifiques (AS/F5/01). Bruxelles (Belgique): Institut pour un développement durable, 2003.

Chia E, Mathé S, Rey-Valette H. Innover en aquaculture pour mettre en place le développement durable. Communication au colloque "Facilitating sustainable innovations: sustainable innovation as a tool for regional development ", Leeuwarden, (Pays-Bas) 26-28 June 2008

Chia E. Principes, méthodes de la recherche en partenariat: une proposition pour la traction animale. Rev Elev Med Vet Pays Trop 2004; $57: 233-40$.

CIPA/ITAVI. IDaqua 2006-2007. L'actualité du programme d'indicateurs de durabilité pour I'aquaculture. Note d'information $\mathrm{n}^{\circ} 1$. Paris: CIPA : ITAVI, 2007.
Droz Y, Lavigne JC. Éthique et développement durable. Genève ; Paris : IUED; Karthala, 2006.

Guinée JB, Gorrée M, Heijungs R, et al. Handbook on life cycle assessment. An operational guide to the ISO standards. Dordrecht (PaysBas) : Kluwer Academic Publishers, 2002.

Godard O, Hubert B. Le développement durable et la recherche scientifique à I'Inra. Rapport à madame la directrice générale de I'Inra. Rapport intermédiaire de mission. Paris: Inra, 2002

ISO (The International Organization for Standardization). Environmental management Life cycle assessment-Principles and framework. ISO 14040. Genève: ISO, 2006.

ISO (The International Organization for Standardization). Environmental management Life cycle assessment - Requirements and guidelines. ISO 14044. Genève : ISO, 2006.

Laloë F. Information at the interface between supply and demand for indicators: the use of a model with estimated parameter values as a base for indication in multi-criteria analyses. Int J Sustainable Dev 2007 ; 10 : 33-45.

Lazard J, Baruthio A, Mathé S, et al. Adaptation des typologies d'exploitations aquacoles aux exigences du développement durable. Cah Agric 2009 ; 18 : 199-210. Doi : 10.1684/ agr.2009.0297

Lazzeri Y. Les indicateurs territoriaux de développement durable. Questionnements et expériences. Paris : L'Harmattan, 2006.

Liu M. Fondements et pratiques de la recherche-action. Paris : L'Harmattan, 1997.

Mathé S, Rey-Valette H, Chia E. Le partenariat d'exploration: une première étape pour la mise en place du développement durable en aquaculture. Poster au 8th European IFSA Symposium "Empowerment of the rural actors: a renewal of farming systems perspectives ", Clermont Ferrand (France), 6-10 July 2008

Mathé S, Brunel O, Rey-Valette $\mathrm{H}$, Clément $\mathrm{O}$. Recensement des initiatives en faveur de la durabilité de I'aquaculture. Rapport. Montpellier (France) : CEP/UICN, 2006.
Offredi C. Introduction générale. In : Offredi C, ed. La dynamique de I'évaluation face au développement durable. Paris : L'Harmattan, 2006.

Prabhu R, Colfer C, Dudley R. Directives pour le développement, le test et la sélection de critères et indicateurs pour une gestion durable des forêts. Montpellier : Cirad, 2000.

Reix R. Système d'observation et management des orgnisations. $5^{\mathrm{e}}$ édition. Coll. Gestion. Paris : Vuibert, 2007

Rey-Valette $\mathrm{H}$, Clément $\mathrm{O}$, Mathé S, Lazard J, Chia E. Un cadre pour analyser le développement durable des systèmes aquacoles littoraux. Communication au colloque international pluridisciplinaire: "Le littoral Subir, Dire, Agir ", Lille 16-18 janvier 2008a.

Rey-Valette $\mathrm{H}$, Clément O, Mathé S, Chia $\mathrm{E}$, Lazard J. Le choix des principes, critères et indicateur de développement durable de l'aquaculture: étapes et condition de l'appropriation du développement durable. Communication au colloque international: "Instituer le développement durable. Appropriation, professionnalisation, standardisation ", Lille 8-10 Novembre 2007a.

Rey-Valette H, Laloë F, Le Fur J. Introduction to the key issue concerning the use of sustainable development indicators. Int J Sustainable Dev $2007 b ; 10: 4-13$.

Rey-Valette H, Clément O, Aubin J, et al. Guide de co-construction d'indicateurs de développement durable en aquaculture. Montpellier (France) : Cirad, 2008b.

Rudlof F. Indicateurs de développement durable et processus de modernisation. In: Lazzeri $\mathrm{Y}$, ed. Les indicateurs territoriaux de développement durable. Questionnements et expériences. Paris : L'Harmattan, 2006.

Segrestin B. La gestion des partenariats d'exploration: spécificités, crises et formes de rationalisation. Thèse, École des mines de Paris, 2003.

UE (Union européenne). Stakeholder Workshop to develop sustainability indicators. www.euraquaculture.info/index.php?option=com. 